\title{
Neuropatia do pé diabético em usuários de uma unidade de saúde da família
}

\begin{abstract}
RESUMO | Caracterizar a presença da neuropatia diabética em usuários diabéticos de uma Unidade de Saúde da Família (USF). Estudo descritivo, exploratório, de caráter quantitativo, desenvolvido em uma USF de Olinda/PE. A amostra foi composta por 106 usuários diabéticos cadastrados na respectiva USF. Foi realizado testes com o apoio de instrumentos como o monofilamento de $10 \mathrm{~g}$ e o teste da sensação de picadas, além de formulário específico. Do total, 85,9\% dos usuários referiram sintomas neuropáticos como dormência e formigamentos e $67,9 \%$ estavam com os pés ressecados. Perante a avaliação vascular, 52,84\% dos avaliados apresentaram o pulso pedioso diminuído. Quanto a avaliação neurológica, 19,81\% dos diabéticos apresentaram alterações na sensibilidade tátil à pressão. Calos estavam presentes em $45,2 \%$ dos avaliados. Foi possível verificar que altas taxas de sinais e sintomas neuropáticos estavam presentes nos diabéticos participantes deste estudo. Desta forma, intervenções precoces precisam ser implementadas na unidade de saúde.
\end{abstract}

Palavras-chaves: Atenção Primária à Saúde; Incidência; Promoção da Saúde.

ABSTRACT | To characterize diabetic neuropathy in diabetic patients in a Family Health Unit (FHU). A descriptive, exploratory, quantitative study developed in a FHU in the Olinda / PE. The sample consisted of 106 diabetic patients enrolled in their FHU. Tests were carried out with the support of instruments such as $10 \mathrm{~g}$ monofilament and the pricking sensation test, besides specific forms. Of the total, $85.9 \%$ of the users reported neuropathic symptoms such as numbness and tingling, and $67.9 \%$ had dry feet. Throughout the vascular evaluation, $52.84 \%$ of the patients presented decreased pedal pulse. As for the neurological evaluation, $19.81 \%$ revealed alterations in the tactile sensitivity to pressure. Callus were present in $45.2 \%$ of the evaluated ones. It was possible to observe high rates of neuropathic signs and symptoms in each one of the participants submitted. Therefore, measures need to be taken in order to implement early interventions in the health unit.

Keywords: Primary health care; Incidence; Health promotion.

RESUMEN | Caracterizar la presencia de la neuropatía diabética en usuarios diabéticos de una Unidad de Salud de la Familia (USF). Estudio descriptivo, exploratorio, de carácter cuantitativo, desarrollado en una USF, Olinda / PE. La muestra fue compuesta por 106 usuarios diabéticos registrados en la respectiva USF. Se realizaron pruebas con el apoyo de instrumentos como el monofilamento de $10 \mathrm{~g}$ y la prueba de la sensación de picaduras, además de formulario específico. Del total, 85,9\% de los usuarios refirieron síntomas neuropáticos como adormecimiento y hormigueos y el $67,9 \%$ estaban con los pies resecados. Ante la evaluación vascular, el $52,84 \%$ de los evaluados presentaron el pulso pedioso disminuido. En cuanto a la evaluación neurológica, el 19,81\% de los diabéticos presentaron alteraciones en la sensibilidad táctil a la presión. Los calos estaban presentes en el 45,2\% de los evaluados. Fue posible verificar que altas tasas de signos y síntomas neuropáticos estaban presentes en los diabéticos participantes de este estudio. De esta forma, las intervenciones tempranas necesitan ser implementadas en la unidad de salud.

Descriptores: Atención primaria a la salud; incidencia; Promoción de la salud.

\section{José William Araújo do Nascimento}

Graduando de Enfermagem da Universidade Católica de Pernambuco.

\section{Suzane Brust de Jesus}

Especialista em Saúde Pública pela Fiocruz em Saúde da Família. Mestre em Linguística pela Universidade Federal da Paraíba. Docente/pesquisadora do Centro de Ciências Biológicas e da Saúde da Universidade Católica de Pernambuco (UNICAP) nos cursos de Enfermagem e Medicina.

\section{Edjôse Ciríaco Santana Silva}

Graduando de Enfermagem da Universidade Católica de Pernambuco.

\section{Manoel Luiz Ferreira Junior}

Graduando de Medicina da Universidade Católica de Pernambuco.

\section{Avanilde Paes Miranda \\ Mestre em Hebiatria - Determinantes de Saúde na Adolescência. Especialista Vigilância Sanitária e Epidemiológica. Especialista Estratégia Saúde da Família. Especialista Docência na Área: Enfermagem, Especialista Gestão em Emergência do SUS. Especialista UTI e Emergência.}

Recebido em: 26/05/2019

Aprovado em: 25/07/2019
INTRODUÇÃO

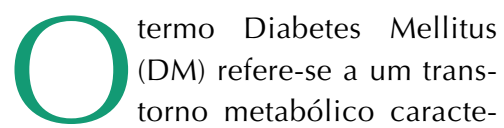

rizado por hiperglicemia e distúrbios no metabolismo de carboidratos, proteínas e gorduras, resultantes de problemas na secreção ou deficiência da secreção e/ ou da ação da insulina ${ }^{1}$. DM é um problema de saúde associado ao estilo de vida de seu portador e do bom manejo deste problema ainda na Atenção Básica $^{2}$. A prevalência do DM nos países da América Central e do Sul é estimada em 26,4 milhões de pessoas e projetada para 40 milhões, em $2030^{3}$. Neste contexto, é utilizado o termo, o pé diabético. Este se caracteriza por lesões cutâneas e profundas relacionadas a al- 
terações neurovasculares, ortopédicas e infecciosas do pé do diabético ${ }^{4}$.

A neuropatia diabética adquire caráter especial uma vez que associada ao componente isquêmico torna o paciente mais vulnerável a infecções nos pés ${ }^{5}$. Esta complicação leva a uma perda da sensibilidade, gerando deformidade do pé, tornando o paciente vulnerável a pequenos traumas que podem precipitar uma ferida, com posterior infecção e evolução a úlcera com consequente amputação do respectivo membro ${ }^{1,4}$. Mais de um milhão de portadores de diabetes sofrem amputação de membro todo ano, como consequência dessa condição ${ }^{6}$.

Os principais sinais e sintomas da neuropatia diabética são dormência e formigamento. Esta complicação ocorre devido à perda axonal ou desmielinização segmentar dos nervos, que ocasiona uma diminuição ou perda severa dos potenciais de ação dos nervos periféricos com consequente retardo na velocidade de condução dos estímulos sensitivos $^{7}$. Desta forma, o paciente com alterações significativas na sensibilidade é propício a ocorrência de traumas repetitivos que podem resultar em ferimentos importantes em membros inferiores (MMII) ${ }^{4}$.

Ao exame físico, pode estar presente a diminuição ou ausência da sensibilidade tátil e dolorosa, a atrofia da musculatura intrínseca dos pés e ressecamento com consequente rarefação dos pelos ${ }^{4,7}$. Os exames neurológicos em pés dos diabéticos, recomendados pelo Ministério de Saúde, incluem a avaliação da sensibilidade tátil à pressão com o monofilamento de Semmes-Weinstein de $10 \mathrm{~g}$, o teste com o diapasão de $128 \mathrm{~Hz}$, o teste para sensação de picadas e o teste para o reflexo aquileu. Para critérios diagnósticos, a utilização do monofilamento de $10 \mathrm{~g}$ acompanhado de qualquer outro teste mencionado, é o suficiente para classificar pacientes em neuropáticos ${ }^{8}$. O Consenso Internacional sobre o Pé Diabético estabelece que a ausência do sentir do monofilamento de $10 \mathrm{~g}$ indica a presen- ça de neuropatia diabética. Também são classificados como portadores da neuropatia autonômica na presença de dois ou mais testes alterados ${ }^{9}$.

Por isso, a detecção e controle das complicações do DM devem ser realizadas em tempo oportuno, com definição de responsabilidades compartilhadas entre a Atenção Básica e os demais níveis de atenção, para acompanhamento e seguimento do caso ${ }^{9}$.

Diante do exposto, teve-se como questão de pesquisa: qual a prevalência da neuropatia diabética em usuários diabéticos de uma Unidade de Saúde da Família (USF)? Assim, este estudo teve como objetivo, caracterizar a presença de pés neuropáticos em usuários diabéticos a fim de auxiliar a equipe e gestores a entender a dimensão do problema que atinge a população de diabéticos.

\section{MÉTODO}

O presente estudo descritivo exploratório de caráter quantitativo foi realizado no município de Olinda, Região Metropolitana do Recife. A pesquisa foi realizada na Unidade de Saúde da Família (USF) do território adscrito de Cohab Peixinhos, que tem aproximadamente 180 diabéticos cadastrados. Destes, 106 participaram do estudo. Foram incluídos todos os portadores de DM cadastrados na respectiva USF, e excluídos todos os diabéticos não cadastrados e os que se recusaram a participar da pesquisa.

A pesquisa foi realizada no período de 6 meses (setembro/2017 a janeiro/2018) na USF avaliada. Após a assinatura do Termo de Consentimento Livre Esclarecido (TCLE), os usuários responderam a um check-list elaborado pelo pesquisador.

Foram realizadas avaliações cutâneas, musculoesqueléticas, vascular e neurológica periférica nos pés dos diabéticos, utilizando-se propedêuticas como a inspeção e palpação. O estado vascular foi aquilatado através de palpações superficiais e profundas dos pulsos tibial posterior (PTP) e pulso pedioso (PP).

A condição neurológica foi averiguada através do estímulo sensitivo tátil à pressão e o doloroso. Para esta primeira utilizou-se o teste de Semmes-Weinstein - monofilamento de $10 \mathrm{~g}$, aplicado no paciente com os olhos fechados, tendo como referência um mapa sensitivo de 4 pontos. A sensibilidade à pressão foi considerada anormal na presença de duas respostas incorretas pelo usuário. Para verificar a sensibilidade dolorosa, utilizou-se o teste de sensação de picadas, onde usou-se um objeto pontiagudo na superfície dorsal da pele próxima a unha do hálux. Foram considerados resultados anormais, a não percepção de pelo menos um estímulo doloroso.

É necessário destacar que foram utilizadas comunicações com médicos e enfermeiros da USF com o intuito de confirmar em alguns usuários, alguns itens presentes no check-list. Todos os usuários que ao exame, portavam alguma sintomatologia de suspeita da neuropatia diabética, foram encaminhados imediatamente as consultas de enfermagem e médica da referida unidade.

Após a coleta de todos os dados, as respostas foram analisadas e consolidadas pelos pesquisadores, tabulados em valores absolutos e percentuais pelo programa de computador Microsoft Excel. Utilizou-se o SPSS (Statistical Package for Social Science) para análise estatística dos dados. A privacidade e a confidencialidade foram asseguradas em todas as fases da pesquisa por meio de codificação. Seguiu-se os preceitos éticos da Resolução n 466 de 12 de dezembro de $2012^{10}$. O projeto foi apreciado pela Comissão de Ética em pesquisa da Universidade Católica de Pernambuco, CAAE: 413440-MED-017-2016/2-2. CAAE Plataforma Brasil 65743417.8.0000.5206 com Parecer Comitê de Ética e Pesquisa 2.009.766.

\section{RESULTADOS}

Os usuários diabéticos foram ini- 
cialmente caracterizados quanto a idade e sexo. Destes, a média de idade dos participantes do estudo foi de 63,6 anos (desvio padrão: 11,2), sendo a maioria do sexo feminino $(80 \%)$.

Conforme mostra a tabela 1,0 maior índice de anormalidades foi o mau controle glicêmico, $80,1 \%$, seguido da pele ressecada $(67,9 \%)$, enquanto $19,8 \%$ já havia possuído algum tipo de úlcera recentemente nos pés. A média do tempo de diabetes entre os usuários que já portavam úlceras diabéticas era de 14,5 anos.

O gráfico 1 mostra os dados referentes a avaliação vascular. Observa-se que $50,48 \%$ dos diabéticos apresentaram pulsações diminuídas e em 5,66\% não havia sequer a presença de pulsações em MMII.

Dentre os 106 diabéticos avaliados, 85,9\% referiram sentir dormência e formigamentos nos membros inferiores. Os resultados da avaliação neurológica periférica (sensibilidade à pressão) dos pés dos usuários avaliados foram expressos no gráfico 2. De acordo com este gráfico, 19,81\% dos avaliados apresentaram ausência da sensibilidade tátil à pressão seja no pé direito ou esquerdo. No que tange a sensibilidade dolorosa, o gráfico 3 expressa a distribuição percentual referente ao teste de sensação de picadas. Observa-se neste gráfico, que 14 usuários (13,21\%) não apresentavam o estímulo doloroso frente ao teste clínico.

Gráfico 3: Distribuição percentual da avaliação da sensibilidade dolorosa em pés de usuários diabéticos de uma USF, Olinda/PE, 2018.

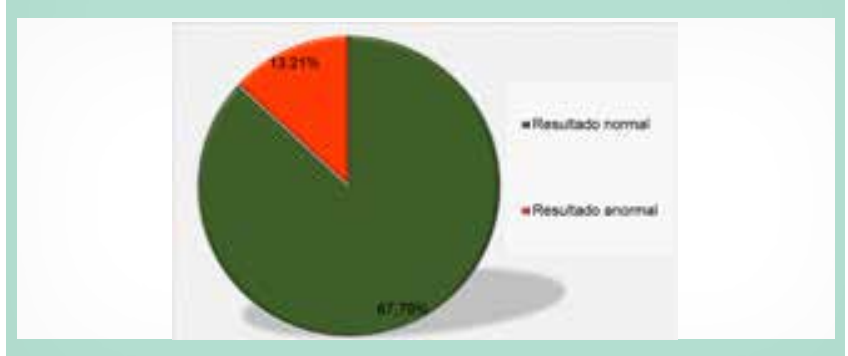

Fonte: Dados da pesquisa

Fonte: Dados da pesquisa

Fonte: Dados da pesquisa
Tabela 1: Distribuição percentual dos principais achados da avaliação clinica nos pés de usuários diabéticos de uma USF. Olinda/PE, 2018.

\begin{tabular}{lcc|}
\hline FATORES DE RISCO $(\mathbf{N}=\mathbf{1 0 6})$ & N & $\%$ \\
\hline Amputação prévia & 4 & 3,7 \\
\hline Doença vascular periférica & 32 & 30,1 \\
\hline Úlcera recente em pés & 21 & 19,8 \\
\hline Mau controle glicêmico & 85 & 80,1 \\
\hline Deformidade nos pés & 43 & 44,7 \\
\hline Tabagismo & 25 & 23,5 \\
\hline Presença de intertrigo micótico & 18 & 16,9 \\
\hline Pés ressecados e/ou descamativos & 72 & 67,9 \\
\hline Unhas espessadas & 67 & 63,2 \\
\hline Presença de eritemas & 58 & 54,7 \\
\hline Presença de dedos em garra & 2 & 1,8 \\
\hline Presença de dedos em martelo & 9 & 8,4 \\
\hline Pé de Charcot & 9 & 8,4 \\
\hline Edema & 45 & 42,4
\end{tabular}

Gráfico 2: Distribuição percentual da avaliação neurológica tátil à pressão por meio do monofilamento de $10 \mathrm{~g}$ em pés de usuários diabéticos de uma USF, Olinda/PE, 2018.

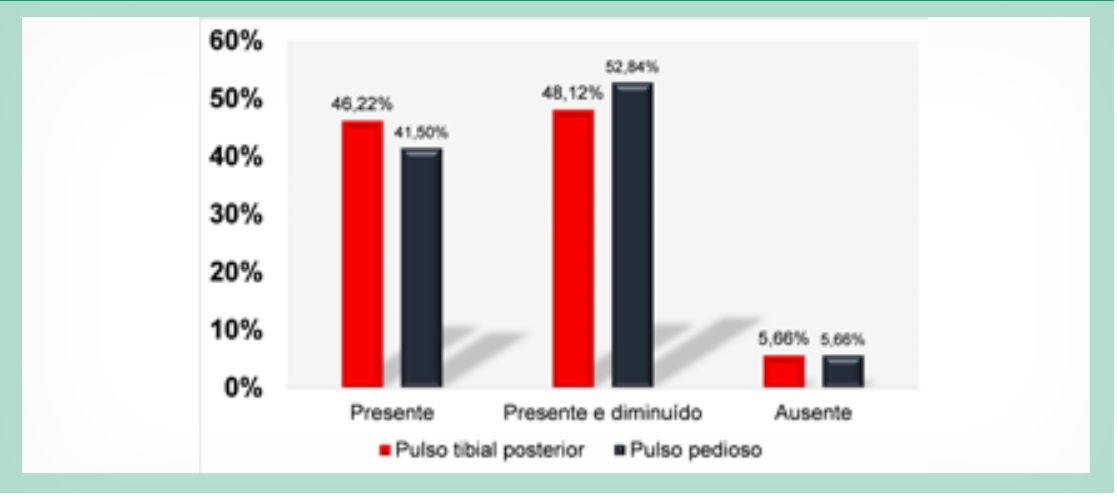

Gráfico 2: Distribuição percentual da avaliação neurológica tátil à pressão por meio do monofilamento de $10 \mathrm{~g}$ em pés de usuários diabéticos de uma USF, Olinda/PE, 2018.

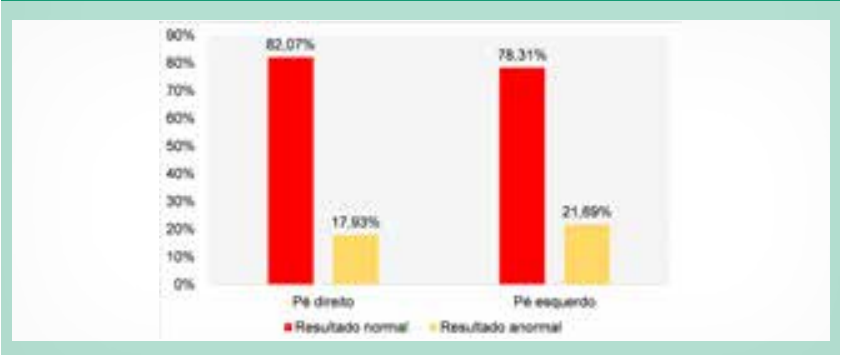

Fonte: Dados da pesquisa 


\section{DISCUSSÃO}

Neste estudo, houve um predomínio de usuários diabéticos do sexo feminino e de indivíduos com idade avançada. O percentual foi expressivo uma vez que essa população procura com mais frequência as unidades de saúde, em especial, a Atenção Primária ${ }^{11}$.

Esse achado foi comparável com um estudo de coorte retrospectivo, onde foi relatado que a idade $\geq 45$ anos é um fator de risco para o desenvolvimento de úlceras do pé diabético ${ }^{12}$.

Em relação aos principais achados da avaliação clínica, verificou-se uma grande taxa de mau controle do diabetes entre os usuários avaliados. A literatura estabelece uma relação exponencial entre este mau controle e as complicações e o tempo de estadia da doença ${ }^{6}$.

A pele ressecada $(67,9 \%)$, foi um sinal clínico importante uma vez que a anidrose e pele seca estão relacionadas a alterações no sistema nervoso neurovegetativo $^{4}$. Se não houver uma prevenção prévia ou um tratamento adequado esse ressecamento pode evoluir a rachaduras e fissuras, caracterizando-se como ponto importante para entrada de microrganismos e infecções subsequentes ${ }^{4,7}$.

Referindo-se a avaliação vascular, o pulso pedioso (PP) obteve a maior taxa de anormalidade (diminuído e ausente), com 58,50\%. É preciso ressaltar que condições como sobrepeso e edema dificultaram a percepção de algumas pulsações. Porém a avaliação da integridade dos pulsos se faz necessária para a identificação precoce do acometimento vascular, uma vez que esta situação é fator de risco para o desenvolvimento de úlceras ${ }^{13}$.

Resultados análogos podem ser observados em uma pesquisa ${ }^{14}$ realizada na cidade do Recife, em que foi possível verificar índices semelhantes, como 12,5\% e $4,2 \%$ relativos a ausências do pulso tibial posterior e PP.

Em relação a avaliação neurológica periférica, faz necessário destacar que $15,65 \%$ dos avaliados na pesquisa apresentaram suspeita diagnóstica, por meio dos testes realizados, de neuropatia periférica. Constatou-se que destes, 78,57\% tinham a pulsação de MMII ausentes ou diminuídas.
Diversas teorias explicam que além do aspecto bioquímico, a microangiopatia afete diretamente a vascularização dos nervos periféricos, tornando o paciente neuropático, devido à perda de função de algumas vias somáticas ${ }^{4}$.

\section{CONCLUSÃO}

Altas taxas de sinais e sintomas neuropáticos foram verificadas nos diabéticos participantes deste estudo. Neste limiar, alguns usuários diabéticos investigados apresentaram alterações da sensibilidade e sintomatologia clínica próprias da presença de neuropatia diabética. Esta, juntamente com os possíveis quadros isquêmicos, podem levar os diabéticos a desenvolverem um quadro de prejuízo anátomo-funcional dos membros inferiores. Foi possível então, alcançar todos os objetivos propostos por esta pesquisa.

É necessário então, que a prevenção e rastreio da neuropatia seja realizada de forma contínua, reforçando a necessidade do diagnóstico precoce, afim de prevenir ulcerações, melhorando a qualidade de vida dos indivíduos diabéticos.

\section{Referências}

1. Katsarou A, Gudbjorndothr S, Rawshani A, Dabelea D, Bonifacio E, Anderson BJ, Jacobsen LM, Schatz DA, Lernmark A. Type 1 diabetes mellitus. Nat Rev Dis Primers. 2017;30(3):17016.

2. Alfradique ME, Bonolo PF, Dourado I, Lima-Costa MF, Macinko J, Mendonça CS, Oliveira VB, Sampaio LFR, Simoni C, Turci MA. Internações por condições sensíveis à atenção primária: a construção da lista brasileira como ferramenta para medir o desempenho do sistema de saúde (Projeto ICSAP - Brasil). Cadernos de Saúde Pública. Rio de Janeiro. 2009:25(6):1337-1349.

3. Organização Mundial da Saúde (OMS). Cuidados inovadores para condições crônicas: componentes estruturais de ação. Brasilia, 2003.

4. Pedrosa HC, Vilar L, Boulton AJM. Neuropatias e pé diabético. 1ed. São Paulo: AC Farmacêutica, 2014.

5. Brinat LM, Diogo NAS, Moreira TR, Mendonça ET, Amaro MOF. Prevalence and factors associated with peripheral neuropathy in individuals with diabetes mellitus. Revista Online de Pesquisa. 2017:9(2):347-355.

6. International Diabetes Federation, IDF Diabetes Atlas, International Diabetes Federation, Brussels, Belgium, 2th edition, 2013.

7. Skyler JS, Bakris GL, Bonifácio E, Darsow T, Eckel RH, Groop L, Handelman Y, Insel RA, Mathieu C, McElvaine AT, Palma JP, Pugliese A, Schatz DA, Sosenko $J M$, Wilding JP, Ratner RE. Differentiation of diabetes by pathophysiology, natural history, and prognosis. Diabetes. 2017;66(2):241-255.
8. Brasil. Ministério da Saúde. Secretaria de Atenção à Saúde. Departamento de Atenção Básica. Cadernos de Atenção Básica, n. 36. Estratégias para o cuidado da pessoa com doença crônica: diabetes mellitus. Brasília, 2013.

9. Consenso Internacional Sobre Pé Diabético. Brasília: Secretaria de Estado de Saúde do Distrito Federal. 2016.

10. Brasil. Ministério da Saúde. Conselho Nacional de Saúde. Resolução 466 de 12 de dezembro de 2012. Diretrizes e normas regulamentadoras para pesquisas envolvendo seres humanos (revoga as seguintes resoluções: 196/96, 404/08 e 303/00). 2012.

11. Peixoto SV, Giatti L, Afradique ME, Lima-Costa MF. Custo das internações hospitalares entre idosos brasileiros no âmbito do Sistema Único de Saúde. Epidemiol. Serv. Saúde. 2004;13(4):239-246.

12. Al-Rubeaan K, Al Derwish M, Ouizi S, Youssef AM, Subhani SN, Ibrahimet $\mathrm{HM}$, et al. Complicações do pé diabético e seus fatores de risco em um grande estudo de coorte retrospectivo. PLoS ONE. 2015;10:e0124446.

13. Sociedade Brasileira de Diabetes (SBD). Diretrizes da Sociedade Brasileira de Diabetes: 2013-2014.São Paulo: AC Farmacêutica, 2014.

14. Bezerra GC, Santos ICRV, Lima JC, Souza MAD. Avaliação do risco para desenvolver pé diabético na atenção básica. Revista da Associação Brasileira de Estomaterapia: estomas, feridas e incontinências (ESTIMA). 2015;13(3). 